Source: Irankhah, S., Ali, A. A., Mallavarapu, M., Soudi, M. R., Subashchandrabose, S., Gharavi, S., \& Ayati, B. (2019). Ecological role of Acinetobacter calcoaceticus GSN3 in natural biofilm formation and its advantages in bioremediation. Biofouling, https://doi.org/10.1080/08927014.2019.1597061

\title{
Ecological role of Acinetobacter calcoaceticus GSN3 in natural biofilm formation and its advantages in bioremediation
}

\author{
Sahar Irankhah ${ }^{\mathrm{a}, \mathrm{b}}$, Ahya Abdi Ali ${ }^{\mathrm{a}}$, Megharaj Mallavarapu ${ }^{\mathrm{b}, \mathrm{c}}$, Mohammad Reza Soudi ${ }^{\mathrm{a}}$, Suresh \\ Subashchandrabose ${ }^{\mathrm{b}, \mathrm{c}}$, Sara Gharavi ${ }^{\mathrm{d}}$ and Bita Ayati ${ }^{\mathrm{e}}$
}

a Department of Microbiology, Faculty of Biological Sciences, Alzahra University, Tehran, Iran; ${ }^{b}$ Global Centre for Environmental Remediation (GCER), Faculty of Science and Information Technology, University of Newcastle, Callaghan, NSW, Australia;

${ }^{\mathrm{c} C R C}$ CARE, Newcastle University LPO, Callaghan, NSW, Australia;

${ }^{\mathrm{d} D e p a r t m e n t ~ o f ~ B i o t e c h n o l o g y, ~ F a c u l t y ~ o f ~ B i o l o g i c a l ~ S c i e n c e s, ~ A l z a h r a ~ U n i v e r s i t y, ~ T e h r a n, ~ I r a n ; ~}$

${ }^{\mathrm{e}}$ Environmental Engineering Division, Civil and Environmental Engineering Faculty, Tarbiat Modares University, Tehran, Iran

\section{ABSTRACT}

This study assessed the role of a new Acinetobacter calcoaceticus strain, GSN3, with biofilm-forming and phenol-degrading abilities. Three biofilm reactors were spiked with activated sludge (R1), green fluorescent plasmid (GFP) tagged GSN3 (R2), and their combination (R3). More than 99\% phenol removal was achieved during four weeks in R3 while this efficiency was reached after two and four further operational weeks in R2 and R1, respectively. Confocal scanning electron microscopy revealed that GSN3-gfp strains appeared mostly in the deeper layers of the biofilm in R3. After four weeks, almost $7.0710^{7}$ more attached sludge cells were counted per carrier in R3 in comparison to R1. Additionally, the higher numbers of GSN3-gfp in R2 were unable to increase the efficiency as much as measured in R3. The presence of GSN3-gfp in R3 conveyed advantages, including enhancement of cell immobilization, population diversity, metabolic cooperation and ultimately treatment efficiency.

KEYWORDS: Acinetobacter calcoaceticus; biofilm-forming bacteria; bioremediation; phenol degradation; removal efficiency improvement 